\title{
Author Correction: III-V-on-silicon solar cells reaching 33\% photoconversion efficiency in two-terminal configuration
}

Romain Cariou (1D, Jan Benick, Frank Feldmann, Oliver Höhn, Hubert Hauser, Paul Beutel, Nasser Razek, Markus Wimplinger, Benedikt Bläsi, David Lackner, Martin Hermle, Gerald Siefer, Stefan W. Glunz, Andreas W. Bett and Frank Dimroth (1)

Correction to: Nature Energy https://doi.org/10.1038/s41560-018-0125-0, published online 2 April 2018.

In the version of this Article originally published, in the 'Rear-side light trapping' paragraph of the Methods section, the values of depth and fill factor were incorrectly given as $350 \mathrm{~nm}$ and 50\%, respectively; instead, the values should have read $250 \mathrm{~nm}$ and $60 \%$. This has now been corrected. 